Published in J. Low Temp. Phys. 138, 543-548 (2005).

\title{
Questions Related to the Oscillatory Flow of He II through a Grid at Low Temperatures
}

\author{
P.C. Hendry ${ }^{1}$, P.V.E. McClintock ${ }^{1}$, H.A. Nichol $^{1}$, \\ L. Skrbek ${ }^{2}$ and W.F. Vinen ${ }^{3}$ \\ ${ }^{1}$ Department of Physics, Lancaster University, Lancaster LA1 4YB, UK. \\ ${ }^{2}$ Joint Low Temperature Laboratory, Institute of Physics ASCR and \\ Charles University, V Holešovičkách 2, 18000 Prague, Czech Republic. \\ ${ }^{3}$ School of Physics and Astronomy, University of Birmingham, \\ Birmingham B152TT, UK
}

The flow of pure He II at low temperatures and a range of pressures is probed using an electrostatically-driven oscillating grid. With increasing oscillation amplitude, a (history dependent) first threshold is reached where the initially pure superflow abruptly changes: the resonant frequency decreases and the response becomes strongly nonlinear, attributable to quantized vortices responding to the motion of the grid so as to increase its effective mass without additional damping. On further increase of oscillation amplitude a second threshold is reached, probably marking the onset of superfluid turbulence. The increase in effective mass is believed to be due to a boundary layer of vortex loops that can evolve into turbulent flow at the second threshold. Open questions and problems for future research are formulated.

\section{INTRODUCTION}

The superfluidity of liquid ${ }^{4} \mathrm{He}$ disappears abruptly when critical velocities are exceeded. It has long been appreciated that this process may involve either the creation of rotons ${ }^{1}$ or quantized vortices ${ }^{2,3}$ or, much more commonly, the expansion of remanent ${ }^{4}$ vortices pre-existing in the liquid. In flow experiments it is the latter process that usually dominates, leading to critical velocities of typically $\mathrm{mm} \mathrm{s}^{-1}$ or $\mathrm{cm} \mathrm{s}^{-1}$. In stationary He II, the remanent vortices are presumed to be pinned between protuberances on the vessel walls. The mechanism through which they suddenly expand out into the flowing liquid to form a tangle of quantum vorticity, once the critical 


\section{P.C. Hendry et al}

velocity has been attained, has been studied for e.g. a micron-sized orifice of a particular shape ${ }^{5}$ but, in general, has remained something of a mystery.

Recent experiments ${ }^{6,7}$ appear, however, to offer the possibility of gaining insight into the fundamental processes in question. They involve studies of He II flow due to a macroscopically large electrostatically-driven oscillating grid in the zero temperature limit where the normal fluid component of He II is almost absent. Electron micrographs show that one side of the grid is relatively smooth, whereas the other is densely covered in approximately hemispherical submicron-sized protuberances ${ }^{8}$. This microscopic surface roughness may perhaps be significant for the interpretation of the results obtained (see below). Where influenced by vorticity, the properties of flow through the grid represent an average over a large ensemble of quantized vortices, and should be reproducible and independent of the positions/configurations of individual vortex lines. Details of the experiments ${ }^{6,7}$ and some aspects of current theoretical understanding ${ }^{8}$ have already been published. In what follows, we attempt to summarise the status of the work and identify the remaining open questions an opportunities for the future.

\section{EXPERIMENTAL OBSERVATIONS}

A striking feature of the observed grid response is that it is historydependent. Fig. 1 shows how the resonance curves differ if measured following an increase of pressure in the cell (we refer to such a response as virgin), as compared to the behaviour after violently shaking the grid by means of the highest available drive $\approx 10 \mathrm{~V}_{\mathrm{pp}}$ (which we refer to as regular). Following this "cleaning" procedure, the resonant frequency and linewidth were reproducible to $\pm 0.1 \mathrm{~Hz}$ on a time scale of days.

Fig. 1 shows that the virgin and regular behaviours differed in three important respects. First, the virgin resonant frequency is slightly downshifted. Secondly, the virgin linewidth at low drive was about $0.5 \mathrm{~Hz}$, compared to $\sim 0.2 \mathrm{~Hz}$ for a "cleaned" grid. Thirdly, as the response amplitude reached about $100 \mathrm{mV}_{\mathrm{pp}}$, the behaviour abruptly changed, in that very robust reproducible beatings were then obtained ${ }^{7}$. Remanent vorticity ${ }^{4}$ provides the most likely explanation of these differences. Increasing the cell pressure presumably causes generation of quantized vortices in the jet from the filling capillary. They are likely to reconnect, mutually and with image vortices in walls and electrodes, leaving additional remanent vorticity pinned to the grid or between the grid and the surrounding electrodes. They may well cause the additional shift in resonant frequency in the low drive limit and broadening of the resonance peak (see Fig. 1). 
Questions Related to the Oscillatory Flow of He II through a Grid...

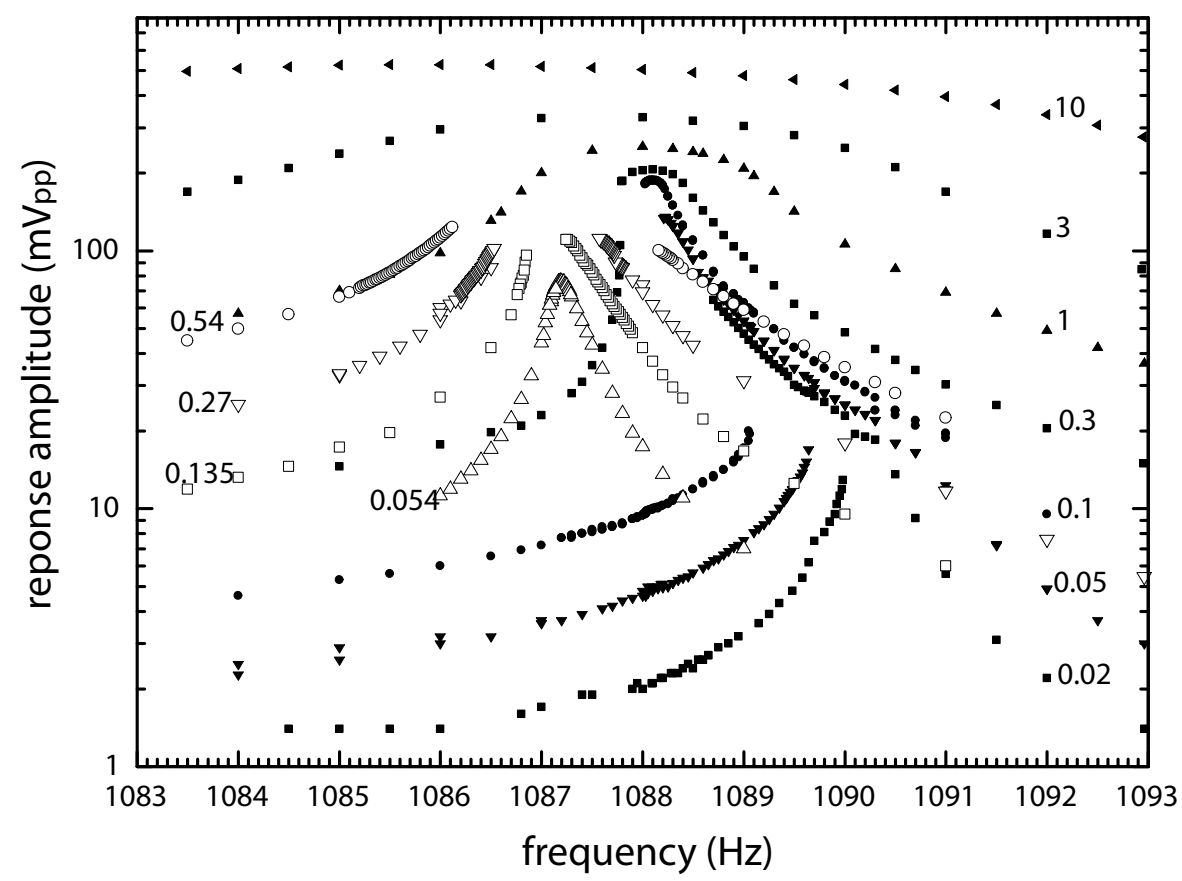

Fig. 1. Resonance curves measured at 3.5 bar using the memory oscilloscope for drive levels (in $\mathrm{V}_{\mathrm{pp}}$ ) as indicated. Filled symbols represent the regular grid behaviour, while the virgin behaviour is represented by resonant curves composed of the open symbols. The upper three curves are shown up to the drive amplitude at which the onset of beating occurred ${ }^{6,7}$.

The regular behaviour of the "cleaned" grid was qualitatively the same at all pressures. For low drives, the resonant response is Lorentzian, with a quality $Q$ factor comparable to that in vacuum. Resonance curves measured in vacuum coincide with those for the same drive levels in He II, provided that the frequency scale is shifted down by about $30 \mathrm{~Hz}$, an effect well understood in terms of the hydrodynamic enhancement of the effective mass of the $\operatorname{grid}^{7}$. As the drive increases, the mean grid velocity reaches a first threshold of $0.3<v_{g}^{(1)}<0.6 \mathrm{~cm} / \mathrm{s}$, beyond which the oscillation amplitude at resonance still continues to rise in proportion to the drive, while the resonant frequency rather suddenly starts decreasing. Stable hysteresis loops are observed; one can circle them many times, providing that the frequency is changed very slowly or in very small steps. They are not appreciably affected by an increase of temperature up to our maximum of $130 \mathrm{mK}$. The data therefore represent phenomena occurring in the zero temperature limit of He II flow. The down-shift in frequency with increasing drive reaches 


\section{P.C. Hendry et al}

typically $2 \mathrm{~Hz}$ (see Fig. 1 ceasing at a second pressure-independent threshold. With further increase in drive, the oscillation amplitude at resonance initially remains almost constant, while the widths of the resonance curves increase rapidly. Only for drive levels exceeding by about an order of magnitude that needed to reach the second threshold does the amplitude at resonance grow again; this time approximately in proportion to the square-root of the drive, typical for a fully developed turbulent flow in a classical liquid. It is therefore most likely that the second threshold marks the onset of turbulence.

\section{THEORETICAL PICTURE}

How can we account for the pronounced downwards frequency shift that occurs while the resonant response rises from the first to the second threshold?

A possible explanation has been formulated ${ }^{8}$ in terms of small loops of remanent vorticity. The surface roughness of the grid (see above) may be an important factor in the pinning of such loops. The response of a vortex loop will depend on whether it is significantly longer or shorter than the minimum resonant length $\ell \approx 13 \mu \mathrm{m}$ required by the dispersion relation for a Kelvin wave. If the loop is shorter than $\ell$, it will respond adiabatically, in that its position and configuration in the flow will always correspond to equilibrium. For loops that are significantly longer than $\ell$, Kelvin waves will be excited, leading to dissipation. It may occur either through to the transfer of energy via a Kelvin wave cascade to very high frequencies, at which there can be significant phonon radiation, or through the evaporation of small vortex rings ${ }^{9}$ ) following reconnection. It is likely that the longer vortex loops (including those connecting the grid to the neighbouring electrodes) are removed during the "cleaning" procedure. When a vortex loop pinned by both ends to the grid responds adiabatically, it enhances the effective mass of the grid by $\varrho_{H e I I} \kappa(d S / d \mathrm{v})$, without increasing the damping ${ }^{8}$. Here $S(\mathrm{v})$ is the area between the loop and the neighbouring walls. For small velocities, $S(\mathrm{v})$ is probably a linear function of the flow velocity, v, and the overall effect is a constant, amplitude independent, enhancement of the effective mass of the grid. If $S(\mathrm{v})$ were to become nonlinear at the first threshold, however, the observed highly nonlinear form of measured resonance curves can be accounted for: it would be necessary for the non-linear part of the loop area

to contribute $\sim 2500 \mu \mathrm{m}^{2}$ per window of the grid at the second threshold. It follows that mass enhancement through this mechanism cannot be due only a few loops - they would be too long to respond adiabatically. If one considers also the stability of these loops against "ballooning out" below the second 


\section{Questions Related to the Oscillatory Flow of He II through a Grid...}

threshold, the analysis ${ }^{8}$ suggests a dense boundary layer constituted of small loops, with $\sim 10^{3}$ per grid aperture, i.e., at least $\sim 10^{8}$ loops altogether, each sized $\sim 1 \mu \mathrm{m}$ or less.

\section{DISCUSSION}

It is tempting to suppose that the formation of an effective boundary layer constituted of vortex loops is universal. It is consistent with our observation that, while increasing the drive amplitude, the system seems to encounter a nucleation problem when passing the first threshold: on some occasions the response stopped growing with increasing drive level, and jumped onto the usual response/drive curve only later. With decreasing drive this feature disappears, and the response remains always proportional to the drive level. Perhaps, employing the ideas of Kusmartsev ${ }^{10}$, the boundary layer can grow from a "plasma" of half vortex rings, although the critical velocities derived by Kusmartsev are larger than those considered here. The difficulties associated with intrinsic vortex nucleation are well-established ${ }^{3}$ and intrinsic critical velocities in microscopically small channels are assumed to be $\sim 30 \mathrm{~m} / \mathrm{s}$ at low temperature. Here, on the other hand, we have an open geometry, the grid surface is very rough and substantial enhancement of flow around excrescences is possible.

Another possibility is that the boundary layer is essentially extrinsic, in that quantized vortex loops are generated on the surface of the grid by macroscopic superflow around it, involving growth from remanent vortex lines ${ }^{4}$, perhaps during the cleaning procedure.

It is interesting to characterize the first threshold in terms of a peak superfluid Reynolds number $R e_{s}=U_{\max } G / \kappa \approx 10-30$, where $U_{\max }$ stands for a peak critical flow velocity through the grid window, $G$ is its linear size and $\kappa$ denotes the circulation quantum. The observed values of $R e_{s}$ compare surprisingly well with the critical $R e_{s}=U D / \kappa \approx 20$ ( $U$ is the transport velocity and $D$ the diameter of the pipe) found as a temperature independent threshold in pipe flow of He II at much higher temperatures when the flow of the normal component was inhibited by superleaks placed at both ends of the pipe ${ }^{11}$. This suggests that the underlying physical mechanism, although not understood, might persist at temperatures above $1.4 \mathrm{~K}$ where there is an appreciable fraction of normal fluid.

There is experimental evidence that the position of the first threshold might depend on the highest drive previously used, i.e., on the degree of "cleaning" ${ }^{-8}$. This observation supports the idea that driving the grid at high amplitude causes a re-arrangement of remanent vorticity. 


\section{P.C. Hendry et al}

\section{OPEN QUESTIONS}

The results described pose intriguing fundamental questions, such as: (i) Is the formation of an effective boundary layer constituted of vortex loops intrinsic to any temperature and any superflow over a solid boundary? (ii) Is the nucleation process of vortex loops of intrinsic or extrinsic origin? (iii) How is the nucleation process affected by frequency, surface roughness, history, temperature? (iv) Why is the first threshold pressure dependent, while the second seems not? (v) Can we learn more about generation of quantum turbulence, or, possibly, turbulence in general? (vi) Are the results exclusive to pure He II, or can they also be observed in boundary layer flows of other quantum fluids? It is the extreme lightness of the grid that promises to facilitate investigation of the growth of the loops from remanent vortices - for most materials/structures, the corresponding changes in effective mass would be unobservably small. We hope that such studies will lead to a deeper understanding of this process, which is fundamental to the breakdown of superfluidity in most situations of practical interest.

It is a pleasure to acknowledge fruitful discussions with many colleagues, especially D. Charalambous, A.M. Guénault, M. Krusius, and M. Tsubota. The research was supported by the Engineering and Physical Sciences Re-

search Council (UK) and by the Czech Grant Agency under grant No. GAČR $202 / 02 / 0251$.

\section{REFERENCES}

1. T. Ellis and P. V. E. McClintock, Phil. Trans. R. Soc. (Lond.) A 315, 259 (1985).

2. P. C. Hendry, N. S. Lawson, P. V. E. McClintock, and C. D. H. Williams, Phys. Rev. Lett. 60, 604 (1988).

3. E. Varoquaux and O. Avenel, Phys. Rev. B 68, 054515 (2003).

4. D. D. Awschalom and K. W. Schwarz, Phys. Rev. Lett. 52, 49 (1984).

5. K. W. Schwarz, Phys. Rev. Lett. 71, 259 (1993).

6. H. A. Nichol, L. Skrbek, P. C. Hendry, and P. V. E. McClintock, Phys. Rev. Lett., in press .

7. H. A. Nichol, L. Skrbek, P. C. Hendry, and P. V. E. McClintock, submitted to Phys. Rev. E. .

8. W. F. Vinen, L. Skrbek, and H. A. Nichol, J. Low Temperature Phys. (in press) (2004).

9. C. F. Barenghi and D. Samuels, Phys. Rev. Lett. 89, 155302 (2002).

10. F. V. Kusmartsev, Phys. Rev. Lett. 76, 1880 (1996).

11. M. L. Baehr, L. B. Opatowsky, and J. T. Tough, Phys. Rev. Lett. 51, 2295 (1983). 